\title{
Glycemic control and adipokines after periodontal therapy in patients with Type 2 diabetes and chronic periodontitis
}

\author{
Shunqin WANG(a) \\ Jingsong $\operatorname{LIU}^{(b)}$ \\ Junfeng ZHANG ${ }^{(b)}$ \\ Jiancheng $\operatorname{LIN}^{(\mathrm{c})}$ \\ Shuyu YANG (d) \\ Jiangwu $Y^{\prime} O^{(e)}$ \\ Minquan DU(a) \\ (a)Wuhan University, School \& Hospital of \\ Stomatology, Wuhan, China. \\ (b)Xiamen Lianqian Community Health Center, \\ Xiamen, China \\ (c)Xiamen Children's Hospital, Xiamen, China \\ (d) Hospital of Xiamen University, Xiamen, China \\ (e)Xiamen Stomatological Research \\ Institute, Department of Oral Biology and \\ Biomaterial, Xiamen, China
}

Declaration of Interests: The authors certify that they have no commercial or associative interest that represents a conflict of interest in connection with the manuscript.

\section{Corresponding Author:}

Minquan Du

E-mail: duminquan@whu.edu.cn

hitps://doi.org/10.1590/1807-3107BOR-2017.vol31.0090

Submitted: June 21, 2017

Accepted for publication: Sep 11, 2017

Last revision: Sep 25, 2017

\begin{abstract}
The mechanism by which chronic periodontitis $(\mathrm{CP})$ affects type 2 diabetes (T2DM) remains unclear. Therefore, the aim of this study is to evaluate the effects of periodontal therapy (PT) on the glycemic control and adipokines of patients with T2DM and CP with the purpose of elucidating the possible mechanisms by which CP influences T2DM. Forty-four patients with T2DM and $\mathrm{CP}$ were randomly divided into two groups according to whether they underwent PT. Periodontal status, blood glucose, and the levels of serum tumor necrosis factor $\alpha$ (TNF- $\alpha$ ), interleukin-6 (IL-6), adiponectin (APN), and fibroblast growth factor-21 (FGF-21) were measured at baseline and after 3 months. The results revealed that the probing depth (PD) and attachment loss (AL) were significantly improved, the serum levels of TNF- $\alpha$ and IL- 6 were significantly decreased, and APN and FGF-21 exhibited substantial increases in the intervention group after 3 months $(p<0.05)$, whereas no significant changes were observed in the control group. The glycated hemoglobin (HbA1c) levels in both groups decreased significantly after 3 months compared with baseline ( $p<0.05)$, but the intervention group exhibited a significantly greater change $(p<0.05)$. In conclusion, PT may relieve periodontal inflammation, which causes a reduction of insulin-antagonizing adipokines and an increase in insulin-sensitizing adipokines, thereby eliciting an improvement in glycemic control.
\end{abstract}

Keywords: Adipokines; Diabetes Mellitus; Periodontitis.

\section{Introduction}

Diabetes mellitus (DM) is a metabolic disorder that is mainly characterized by hyperglycemia due to impaired insulin action. The most common form of DM is type 2 diabetes mellitus (T2DM), which accounts for approximately $90 \%$ of all DM patients. ${ }^{1,2}$ Due to social mobility and rapid urbanization, the global prevalence of DM has increased sharply in recent years. Globally, 415 million adults have diabetes, and by 2040, this number will rise to 642 million. ${ }^{2}$ China now has the largest epidemic worldwide, and a recent study demonstrated that the prevalence of DM is up to $11.6 \%$ (95\% CI: $11.3 \%-11.8 \%$ ) among Chinese adults. ${ }^{3}$

Chronic periodontitis (CP) is one of the most common chronic infectious diseases and is characterized by destruction of the supporting structures of the teeth. According to the estimated data by Paul IE, $46 \%$ of adults in 
the United States have periodontitis, and $8.9 \%$ have severe periodontitis. ${ }^{4}$ In China, periodontal diseases have become one of the major public health problems. The third national epidemiological investigation on oral diseases in Chinese people demonstrated that gingival bleeding and periodontal pocket depths (PDs) $>4 \mathrm{~mm}$ are present in most middle-aged people $(77.3 \%$ and $41.0 \%$, respectively) and many elderly people $(68.0 \%$ and $52.3 \%$, respectively). ${ }^{5}$

Evidence indicates a "two-way" relationship between T2DM and CP. ${ }^{6,7,8,9}$ A large number of epidemiological studies have demonstrated that T2DM may increase the risk of $\mathrm{CP}$ by two- to three-fold. ${ }^{10,11,12}$ Conversely, CP may be a risk factor for worsened glycemic control in diabetic individuals and may increase the risk of related complications. ${ }^{13,14}$ Currently, the mechanism by which T2DM influences $\mathrm{CP}$ is relatively clear, but the direct effect of $\mathrm{CP}$ on T2DM is not yet well understood. ${ }^{9}$ In recent years, the roles of adipokines in inflammatory responses and insulin resistance (IR) have attracted much attention from researchers. Many studies have found that adipose tissue is not only a passive energy reservoir but is also an active endocrine organ that can produce and release diverse secretory proteins called adipokines into the systemic circulation. ${ }^{15}$ Currently, increasing numbers of adipokines are being found to influence inflammatory responses and IR. According to their effects on IR, adipokines can be classified into two categories. The first is insulin-sensitizing adipokines, which can improve insulin sensitivity, including visfatin, adiponectin (APN), fibroblast growth factor-21 (FGF-21), etc. The second is insulin-antagonizing adipokines, which can promote insulin resistance including tumor necrosis factor $\alpha$ (TNF- $\alpha$ ), interleukin-6 (IL-6), resistin, ${ }^{16}$ etc.

Studies suggest CP can lead to an elevated inflammatory state and affect the levels of serum adipokines and inflammatory mediators. ${ }^{17}$ In this study, we investigated the effects of periodontal therapy (PT) on periodontal status, glycemic control, and adipokines in patients with T2DM and CP. Our hypothesis is that $\mathrm{CP}$ increases the levels of systemic inflammatory factors, which can affect serum adipokine levels and act to aggravate insulin resistance and worsen glycemic control. Alternatively, PT and the removal of the cause of this local chronic inflammation may positively influence inflammatory responses and IR by decreasing the levels of insulin-sensitizing adipokines and the increasing the levels of insulin-sensitizing adipokines, which would then improve glycemic control in these patients. Therefore, the aim of this study is to evaluate the effects of PT on the adipokines and glycemic control of patients with T2DM and CP with the purpose of exploring the role of adipokines in the possible mechanism of the effect of CP on T2DM.

\section{Methodology}

\section{Subjects}

The process of subject selection and inclusion is illustrated in Figure 1. A total of 155 T2DM patients with stable statuses who were followed up at Xiamen Lianqian Community Health Center after the diagnoses of diabetes at a grade three hospital between June 2014 and December 2014 were screened via a questionnaire investigation and clinical periodontal examination. Forty-four subjects who fulfilled the following inclusion criteria were selected for further study: 1) patients with a diagnosis of T2DM for over one year by a grade three hospital who volunteered for the research; 2) $6.50 \leq \mathrm{Hb}$ Alc $\% \leq 10.00$; 3) chronic periodontitis, $\geq 15$ remaining teeth, and more than $30 \%$ of teeth with probing depths (PDs) $\geq 5 \mathrm{~mm}$ and attachment loss (AL) $>4 \mathrm{~mm}$, or more than $60 \%$ of teeth with a $\mathrm{PD}>4 \mathrm{~mm}$ and an $\mathrm{AL} \geq 3 \mathrm{~mm}$; ) a body mass index $\left.(\mathrm{BMI})<30 \mathrm{~kg} / \mathrm{m}^{2} ; 5\right)$ without periodontal treatment in the previous 6 months; 6 ) without antibiotic or non-steroidal anti-inflammatory drug administration in the 3 months; and 7) without serious systemic diseases or complications.

A group of 44 random numbers was generated using SPSS ver. 17.0 (SPSS, Inc., Chicago, USA). These numbers were used to recruit and blindly randomize 44 subjects to either a control group ( $n=22$ subjects who underwent no intervention) or an intervention group ( $\mathrm{n}=22$ subject who underwent a periodontal intervention) with a 1:1 allocation ratio. Ultimately, a total of 39 of the 44 recruited patients finished the study. Three patients in the intervention group were lost to follow-up because they could not revisit on 


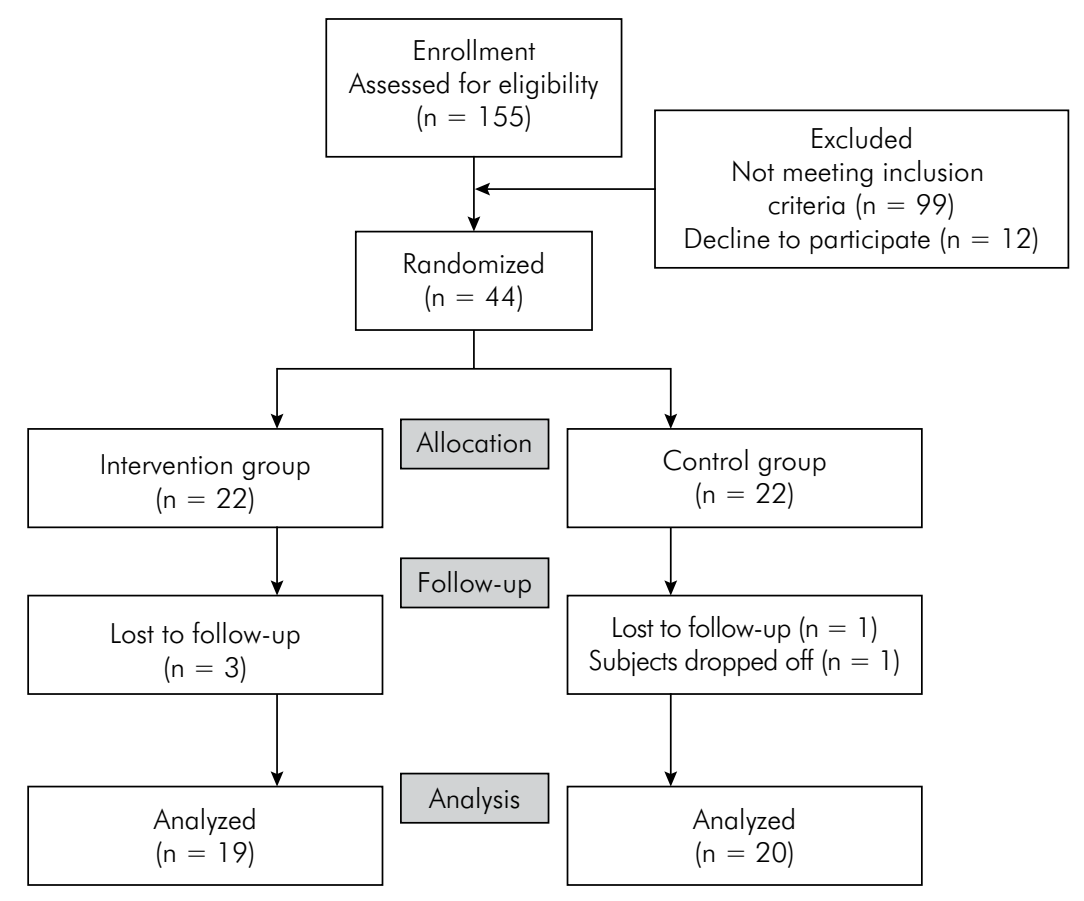

Figure 1. Flow diagram of subject inclusion in the study.

time. One patient in the control group performed the periodontal treatment in the follow-up period, and one patient migrated to another place.

Informed consent was obtained from all patients. This study was approved by the Ethics Committee of Xiamen Dentistry Hospital. To isolate the effects of the periodontal intervention on glycemic control and adipokines, no changes in medications or diet were made for any subject during the study period.

\section{Examiner training and calibration}

To avoid the bias due to different examiners, a single examiner (Jingsong Liu) was involved in the study. The examiner is an attending periodontist and has been treating patients for 8 years in Xiamen Lianqian Community Health Center. Before participating in the study, he had been trained for one month in the Department of Periodontology of Xiamen Dentistry Hospital. To validate the reliability of the periodontal examinations, an intra-examiner reliability assessment was executed, and good agreement (complete agreement rate $>70 \%$ and less than $1 \mathrm{~mm}$ error $>80 \%$ in all parameters) was required.

\section{Clinical periodontal examination}

The PDs and ALs of all of the remaining teeth were tested at baseline and after 3 months. PD and AL were examined at six sites (mesiobuccal, buccal, distobuccal, distolingual, lingual, and mesiolingual) per tooth, and the average was calculated. A Williams periodontal probe was used for the clinical periodontal measurements, and all measurements were performed by a single examiner (Jingsong Liu) who was masked to the systemic status of each patient.

\section{Periodontal intervention}

All subjects in the intervention group underwent a periodontal intervention that included oral hygiene (utilizing correct methods and soft-bristled toothbrushes, interdental brushes and dental floss), full-mouth scaling (supragingival and subgingival scaling), the extraction of hopeless teeth, and the restoration of balanced occlusion. All periodontal interventions were completed by a single periodontist (Jingsong Liu) within two weeks. 


\section{Biological measurements}

Blood samples were collected after overnight fasting, and the sera were obtained by centrifugation at 3,000 revolutions/minute for 10 minutes. The separated serum samples were collected in Eppendorf tubes and stored at $-80^{\circ} \mathrm{C}$ for until further testing. The levels of TNF- $\alpha$, IL- 6 , APN, and FGF21 (test kits from Shanghai Tong Wei Industrial Co., Ltd., China) were tested using enzyme-linked immunosorbent assays (ELISAs) according to standard protocols at baseline and after 3 months. The HbA1c levels were tested with a D-10TM Hemoglobin Analyzer (Bio-Rad Laboratories, Inc., USA).

\section{Statistical analysis}

The statistical analyses were performed with SPSS ver. 17.0. The baseline balance between the groups was determined using independentsamples t-tests or the Pearson $\chi^{2}$ test. One-sample Kolmogorov-Smirnov tests were used to test the distributions of the data for the periodontal parameters, $\mathrm{HbA1c}$, and adipokines. Because the data for the PD, AL, HbA1c, TNF- $\alpha, A P N$ and FGF21 were normally distributed, the differences are presented with bar graphs. The inner-group differences (i.e., baseline vs. after 3 months) were analyzed using paired-sample t-tests, and the intergroup differences were determined using independent-sample t-tests. Because the IL-6 data were not normally distributed, the difference is illustrated with a box plot, and the inner-group differences were analyzed with a non-parametric method (Wilcoxon signed-rank test).

\section{Results}

The demographic variables are presented in Table 1. The study groups were similar in age, gender, degree of education, BMI, the duration of diabetes, smoking, alcohol drinking, and physical exercise ( $p>0.05$ ). The levels of HbA1c, PD, and AL were similar in the study groups at baseline $(\mathrm{p}>0.05)$.

As presented in Table 2, compared with baseline, the levels of PD and AL were significantly improved in the intervention group after 3 months (all $p<0.001)$. The mean changes in the PD and AL were statistically significant in the intervention group after 3 months compared with those in the control group (all $\mathrm{p}<0.001$ ).

At baseline, the HbA1c level exhibited no significant difference between the intervention group and the control group ( $p>0.05)$. After 3 months, a greater reduction in the $\mathrm{HbA1c}$ level was observed in the intervention group, i.e., from $7.63 \pm 0.89$ to $6.99 \pm 0.75$ $(p<0.001)$, whereas a reduction of $7.67 \pm 1.32$ to $7.41 \pm 1.31$ was observed in the control group $(p<0.05)$. When the changes over time were compared between the two groups, the intervention group exhibited a significantly greater change in the $\mathrm{HbA1c}$ level than the control group ( $p<0.05$; Table 2$)$.

As illustrated in Figure 2, the concentrations of serum TNF- $\alpha$ and IL- 6 significantly decreased after periodontal therapy in the intervention group $(p<0.01, p<0.05)$, whereas these concentrations in control group at baseline and at 3 months were not significantly different (both $\mathrm{p}>0.05$; Figure $2 \mathrm{~A}$ and Figure 2B). The APN and FGF21 levels increased significantly in the intervention group after 3 months of the periodontal treatment (both $\mathrm{p}<0.01$ ) but only changed slightly in the control group (both $p>0.05$; Figure 2C and Figure 2D).

\section{Discussion}

Although the association between T2DM and $\mathrm{CP}$ has been extensively discussed, the mechanism by which $\mathrm{CP}$ affects T2DM remains unclear. In the present study, we evaluated the effects of PT on the levels of adipokines and HbA1c in patients with T2DM and CP with the purpose of examining the possible mechanisms by which CP influences T2DM. Four adipokines were selected for study, including two insulin-antagonizing adipokines (TNF- $\alpha$ and IL-6), and two insulin-sensitizing adipokines (APN and FGF21).

The etiology of periodontitis is closely related to dental plaque and calculus deposits in the periodontal tissues. Both healthy individuals and diabetic patients exhibit similar outcomes after periodontal therapy in terms of PD and AL reductions. ${ }^{18,19,20}$ In the present study, we also found that the levels of PD and AL were improved significantly after PT. 
Table 1. Demographic characteristics of the study groups at baseline.

\begin{tabular}{|c|c|c|c|c|}
\hline Characteristic & Intervention group $(\mathrm{n}=19)$ & Control group $(n=20)$ & $t / \chi^{2}$ & $\mathrm{p}$ \\
\hline Age (y) & $61.58 \pm 4.69$ & $61.90 \pm 6.75$ & 0.172 & 0.865 \\
\hline BMI & $24.32 \pm 2.70$ & $23.72 \pm 3.46$ & 0.606 & 0.548 \\
\hline Duration of DM (y) & $8.47 \pm 3.08$ & $7.70 \pm 4.69$ & 1.243 & 0.214 \\
\hline $\mathrm{HbAlc}(\%)$ & $7.63 \pm 0.89$ & $7.67 \pm 1.32$ & 0.119 & 0.906 \\
\hline $\mathrm{PD}(\mathrm{mm})$ & $3.66 \pm 0.60$ & $3.85 \pm 0.58$ & 0.988 & 0.329 \\
\hline $\mathrm{AL}(\mathrm{mm})$ & $4.12 \pm 0.89$ & $4.28 \pm 0.77$ & 0.568 & 0.574 \\
\hline \multicolumn{5}{|l|}{ Gender (n [\%]) } \\
\hline Male & $12(63.16)$ & $14(70.00)$ & 0.205 & 0.741 \\
\hline Female & $7(36.84)$ & $6(30.00)$ & - & - \\
\hline \multicolumn{5}{|l|}{ Education } \\
\hline$\leq$ Primary school & $6(26.32)$ & $3(15.00)$ & 1.703 & 0.427 \\
\hline Middle school & $9(47.33)$ & $13(65.00)$ & - & - \\
\hline$\geq$ College/university & $4(21.05)$ & $4(20.00)$ & - & - \\
\hline \multicolumn{5}{|l|}{ Smoking* (n [\%]) } \\
\hline Yes & $6(31.58)$ & $3(15.00)$ & 1.509 & 0.219 \\
\hline No & $13(68.42)$ & $17(75.00)$ & - & - \\
\hline \multicolumn{5}{|l|}{$\operatorname{Drinking}^{* *}$ (n [\%]) } \\
\hline No & $12(61.15)$ & $17(75.00)$ & 2.838 & 0.242 \\
\hline Seldom & $3(15.79)$ & $2(10.00)$ & - & - \\
\hline Often & $4(21.06)$ & $1(5.00)$ & - & - \\
\hline \multicolumn{5}{|l|}{ Physical exercise ${ }^{* * *}$ (n [\%]) } \\
\hline Seldom & 15 (88.95) & $14(70.00)$ & 0.409 & 0.522 \\
\hline Often & $4(21.05)$ & $6(30.00)$ & - & - \\
\hline \multicolumn{5}{|l|}{ Hypertension $^{* * * *}$ (n [\%]) } \\
\hline Yes & $4(21.05)$ & $8(40.00)$ & 1.642 & 0.200 \\
\hline No & 15 (78.95) & $12(60.00)$ & - & - \\
\hline
\end{tabular}

Table 2. Comparisons of the PD, AL and $\mathrm{HbAlc}$ levels in the two groups after three months.

\begin{tabular}{lcccccc}
\hline \multirow{2}{*}{ Variable } & \multicolumn{3}{c}{ Control group $(\mathrm{n}=20)$} & \multicolumn{3}{c}{ Intervention group (n=19) } \\
\cline { 2 - 7 } & Baseline & After 3 mos & $\Delta$ & Baseline & After 3 mos & $\Delta$ \\
\hline PD & $3.85 \pm 0.58$ & $3.92 \pm 0.56$ & $0.07 \pm 0.28$ & $3.66 \pm 0.60$ & $3.09 \pm 0.63^{b}$ & $-0.57 \pm 0.30^{* *}$ \\
AL & $4.28 \pm 0.77$ & $4.36 \pm 0.81$ & $0.08 \pm 0.32$ & $4.12 \pm 0.90$ & $3.62 \pm 0.84^{b}$ & $-0.50 \pm 0.37^{* *}$ \\
HbAlc & $7.67 \pm 1.33$ & $7.41 \pm 1.32^{a}$ & $-0.27 \pm 0.43$ & $7.63 \pm 0.89$ & $6.99 \pm 0.77^{b}$ & $-0.64 \pm 0.66^{*}$ \\
\hline
\end{tabular}

The results are given as the mean \pm the SD. Intragroup differences were analyzed using paired-sample t-tests. ${ }^{a}: p<0.05 .{ }^{b}: p<0.001$. Intergroup differences were determined using the independent-samples t-test. ${ }^{*}: p<0.05 .{ }^{* *}: p<0.001$. 

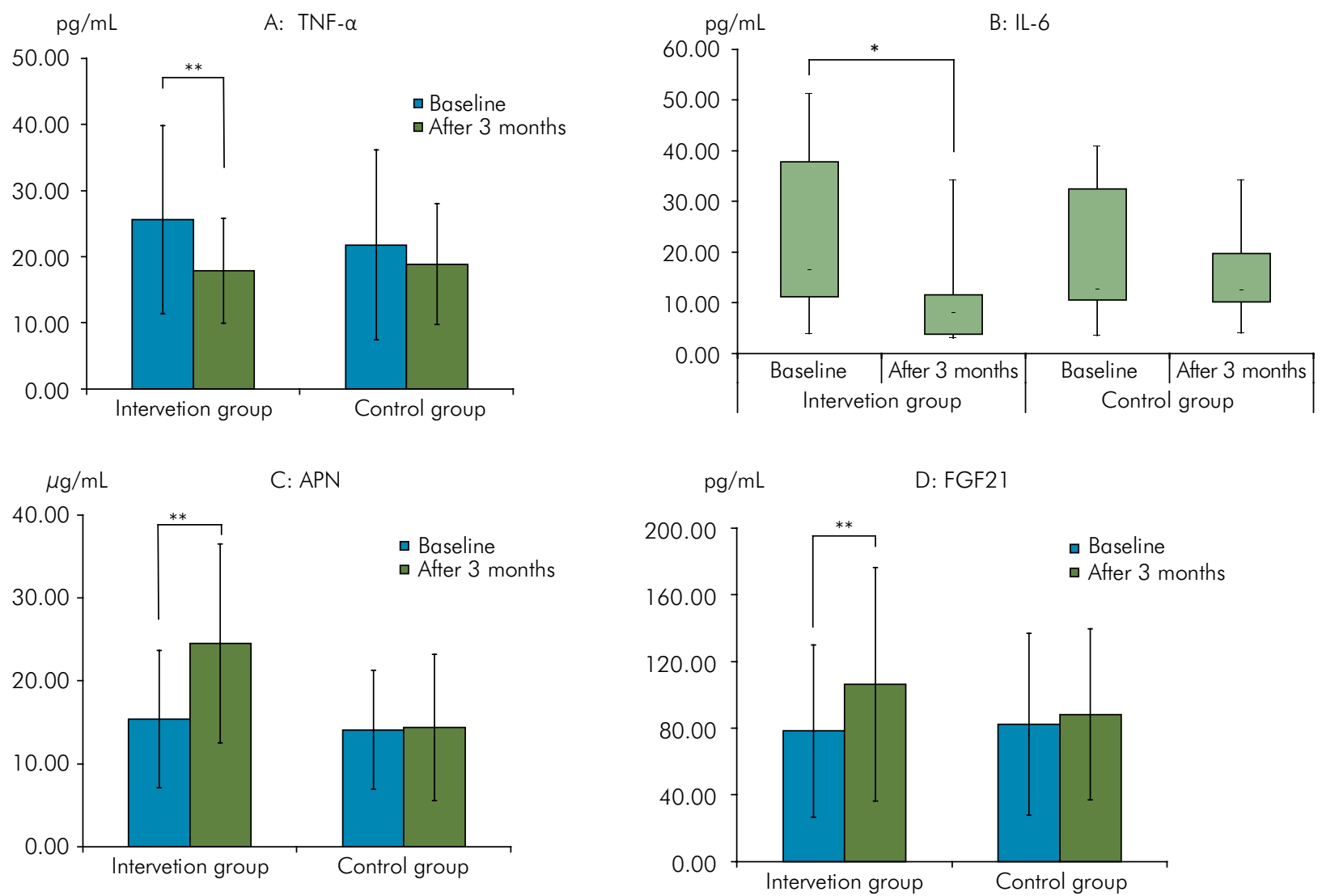

${ }^{*} \mathrm{p}<0.05,{ }^{* *} \mathrm{p}<0.01$

Figure 2. Serum TNF- $\alpha$, IL-6, APN, and FGF21 concentrations in the two groups at baseline and after 3 months.

Therefore, PT, specifically the removal of dental accretions, can relieve the level of periodontal inflammation and improve periodontal conditions.

The HbA1c level reflects the glycemic level over the previous 1 to 3 months. Whether periodontal therapy reduces the $\mathrm{HbA1c}$ level in periodontitis patients remains controversial. Very recently, Teshome $\mathrm{T}$ performed a meta-analysis, and the results revealed a statistically significant $\mathrm{HbA1c}$ reduction of 0.48 (95\%CI: $0.18,0.78)$ in the treatment group compared with the control group ${ }^{21}$, and similar results have been obtained in other studies conducted by Li $\mathrm{Q}^{22}$ and Engebretson $\mathrm{S}^{23}$. In contrast, a multicenter, randomized clinical trial reported that, at 6 months, the mean HbA1c level in the periodontal therapy group increased $0.17 \%$ compared with $0.11 \%$ in the control group with no significant difference between the groups. ${ }^{24}$ Several outcomes of meta-analyses also do not support the notion that PT lowers the level of HbA1c. ${ }^{25,26}$ In the present study, the intervention group exhibited a significantly greater change in the HbA1c level, which indicated that PT may improve glycemic control.

It is believed that pro-inflammatory mediators, such as TNF- $\alpha$ and IL-6, are expressed by the inflamed periodontal sites due to microbial stimuli or a host response. These mediators enter the systemic circulation, interfere with the function of insulin receptors and thereby derange the process of insulin signaling. ${ }^{14} \mathrm{TNF}-\alpha$ was the first inflammatory marker that was thought to play a role in the development of obesity-induced insulin resistance in the 1990s. ${ }^{27}$ TNF- $\alpha$ causes impaired insulin-derived peripheral uptake of glucose by increasing the serine phosphorylation of insulin receptor substrate 1 (IRS-1) and consequently inhibits the translocation of glucose transporter type 4 (GLUT4) to the plasma 
membrane, which results in peripheral IR. ${ }^{28,29}$ Some studies have suggested that treatment with antiTNF- $\alpha$ antibody can improve insulin sensitivity in IR patients ${ }^{30,31}$. IL-6 is another inflammatory adipokine that is involved the pathogenesis of IR. Agarwal A compared 40 patients with impaired fasting glucose (IFG) with 40 healthy people, and IL-6 was found to be significantly elevated in the IFG group and to significantly correlate with IR. ${ }^{32}$ A prospective cohort study conducted by Bertoni $\mathrm{AG}^{33}$ found that serum IL-6 levels are significantly associated with T2DM. IL-6 induces IR through the prevention of non-oxidative glucose metabolism and the suppression of lipoprotein lipase, which increases the triglyceride level. ${ }^{34}$

In the present study, significant reductions in serum TNF- $\alpha$ and IL- 6 after 3 months of therapy were observed. These findings agree with those of other studies. ${ }^{35,36}$ Recently, the outcome of a meta-analysis of 6 randomized-controlled trials and 3 controlled clinical trials revealed a statistically significant mean difference (MD) in TNF- $\alpha(-1.33 \mathrm{pg} / \mathrm{ml}, 95 \% \mathrm{CI}$ : $-2.10 ;-0.56)$ favoring the periodontal intervention versus the control. ${ }^{35}$ In a prospective blind intervention trial, the level of IL-6 exhibited a significant decrease. ${ }^{36}$ These results indicate that PT may relieve periodontal inflammatory conditions and further decrease proinflammatory, insulin-antagonizing adipokines.

APN is a protein that is secreted from adipose tissue and is an insulin-sensitizing, anti-inflammatory adipokine that has been demonstrated to improve glucose tolerance and insulin resistance in humans. ${ }^{37}$ Studies have demonstrated that adiponectin improves insulin sensitivity through the inhibition of TNF$\alpha$-induced adhesion molecule expression and the inhibition of NF- $\kappa \mathrm{B}$ activation. ${ }^{38}$ Many studies have revealed that effective periodontal intervention can increase the serum APN levels in T2DM patients with periodontitis. ${ }^{39,40}$. FGF-21 was discovered to be a potent regulator of glucose uptake in mouse 3T3-L1 cells and primary human adipocytes. ${ }^{41,42}$ FGF-21 can improve insulin sensitivity and lipid metabolism in obese and diabetic animal models and has been proposed as a potential therapeutic agent for the treatment of T2DM, obesity and related complications. FGF-21 significantly improves insulin signaling by enhancing the phosphatidylinositol 3-kinase (PI3K)/AKT pathway, up-regulating glucose uptake, promoting the release of insulin-sensitizing adipokines, such as adiponectin, and reducing the release of insulin-antagonizing leptin..$^{41}$ This study represents the first introduction of FGF21 into a periodontal intervention study that aimed to investigate the effect of PT on FGF21. We found that the serum APN and FGF21 levels were significantly increased after therapy, which indicated that the effective control of inflammation by periodontal treatment may contribute to increasing systemic insulin-sensitizing adipokines, such as APN and FGF21, thus improving the overall health status.

There are several limitations to this study. First, the subjects were elderly; thus, it remains uncertain whether the results of this study can be generalized to other diabetic populations. Second, this study only observed the effect after three months, and the long-term effects after intervention should be considered. Therefore, a larger and long-term epidemiological study should be conducted in the future.

\section{Conclusion}

In summary, PT relieved the periodontal inflammatory status, which in turn caused reductions in insulin-antagonizing adipokines and increases in insulin-sensitizing adipokines that were reflected by an improvement in glycemic control in T2DM patients with CP. Large-scale and long-term human epidemiological studies and animal model studies exploring the role of adipokines in the mechanisms by which CP influences T2DM are necessary.

\section{Acknowledgments}

We thank all of the patients who participated in this study and the nurses in Xiamen Lianqian Community Health Center for their contributions to contacting the patients and the questionnaire survey. This work was supported in part by grants from the Xiamen Health Bureau (grant number: WSK 2012-01) and the National Institute of Hospital Administration (the hierarchical medical treatment policy in diabetes project). 
- Glycemic control and adipokines after periodontal therapy in patients with Type 2 diabetes and chronic periodontitis

\section{References}

1. Afable A, Karingula NS. Evidence based review of type 2 diabetes prevention and management in low and middle income countries. World J Diabetes. 2016;7(10):209-29. https://doi.org/10.4239/wjd.v7.i10.209

2. International Diabetes Federation. IDF diabetes atlas. 7th ed. Local: International Diabetes Federation; ano de publicação.

3. Xu Y, Wang L, He J, Bi Y, Li M, Wang T et al.

Prevalence and control of diabetes in Chinese adults. JAMA. 2013;310(9):948-59.

https://doi.org/10.1001/jama.2013.168118

4. Eke PI, Dye BA, Wei L, Slade GD,

Thornton-Evans GO, Borgnakke WS et al. Update on prevalence of periodontitis in adults in the United States: NHANES 2009 to 2012. J Periodontol. 2015;86(5):611-22. https://doi.org/10.1902/jop.2015.140520

5. Hu DY, Hong X, Li X. Oral health in China: trends and challenges. Int J Oral Sci. 2011;3(1):7-12. https://doi.org/10.4248/IJOS11006

6. Preshaw PM, Alba AL, Herrera D, Jepsen S, Konstantinidis A, Makrilakis $\mathrm{K}$ et al. Periodontitis and diabetes: a two-way relationship. Diabetologia. 2012;55(1):21-31. https://doi.org/10.1007/s00125-011-2342-y

7. Mealey BL, Ocampo GL. Diabetes mellitus and periodontal disease. Periodontol 2000. 2007;44(1):127-53. https://doi.org/10.1111/i.1600-0757.2006.00193.x

8. Casanova L, Hughes FJ, Preshaw PM. Diabetes and periodontal disease: a two-way relationship. Br Dent J. 2014;217(8):433-7. https://doi.org/10.1038/sj.bdj.2014.907

9. Gurav AN. Management of diabolical diabetes mellitus and periodontitis nexus: are we doing enough? World J Diabetes. 2016;7(4):50-66. https://doi.org/10.4239/wjd.v7.i4.50

10. Rajhans NS, Kohad RM, Chaudhari VG, Mhaske NH. A clinical study of the relationship between diabetes mellitus and periodontal disease. J Indian Soc Periodontol. 2011;15(4):388-92. https://doi.org/10.4103/0972-124X.92576

11. Hong M, Kim HY, Seok H, Yeo CD, Kim YS, Song JY et al. Prevalence and risk factors of periodontitis among adults with or without diabetes mellitus. Korean J Intern Med. 2016;31(5):910-9. https://doi.org/10.3904/kjim.2016.031

12. Weinspach K, Staufenbiel I, Memenga-Nicksch S, Ernst S, Geurtsen W, Günay H. Level of information about the relationship between diabetes mellitus and periodontitis: results from a nationwide diabetes information program. Eur J Med Res. 2013;18(1):6-13. https://doi.org/10.1186/2047-783X-18-6

13. Khanuja PK, Narula SC, Rajput R, Sharma RK, Tewari S. Association of periodontal disease with glycemic control in patients with type 2 diabetes in Indian population. Front Med. 2017;11(1):110-9. https://doi.org/10.1007/s11684-016-0484-5

14. Santos Tunes R, Foss-Freitas MC, Nogueira-Filho GR. Impact of periodontitis on the diabetes-related inflammatory status. J Can Dent Assoc. 2010;76:a35.
15. Li Y, Ding L, Hassan W, Abdelkader D, Shang J. Adipokines and hepatic insulin resistance. J Diabetes Res. 2013;2013:170532. https://doi.org/10.1155/2013/170532

16. Zeng J, Yang GY. Recent advances in the study of the relationship and mechanism between the adipocytokines and insulin resistance. J Chendu Med Coll. 2011;6(1):78-82. [Chinese.]

17. Andrukhov $O$, Ulm C, Reischl H, Nguyen PQ, Matejka M, Rausch-Fan X. Serum cytokine levels in periodontitis patients in relation to the bacterial load. J Periodontol. 2011;82(6):885-92. https://doi.org/10.1902/jop.2010.100425

18. Christgau M, Palitzsch KD, Schmalz G, Kreiner U, Frenzel S. Healing response to non-surgical periodontal therapy in patients with diabetes mellitus: clinical, microbiological, and immunologic results. J Clin Periodontol. 1998;25(2):112-24. https://doi.org/10.1111/j.1600-051X.1998.tb02417.x

19. Kara G, Cifcibasi E, Karsidag K, Cintan S. Short term effects of periodontal therapy on inflammatory markers in patients with type-2 diabetes. Saudi Med J. 2015;36(4):469-76. https://doi.org/10.15537/smi.2015.4.10380

20. Hayashi J, Hasegawa A, Hayashi K, Suzuki T, Ishii M, Otsuka $\mathrm{H}$ et al. Effects of periodontal treatment on the medical status of patients with type 2 diabetes mellitus: a pilot study. BMC Oral Health. 2017;17(1):77. https://doi.org/10.1186/s12903-017-0369-2

21. Teshome A, Yitayeh A. The effect of periodontal therapy on glycemic control and fasting plasma glucose level in type 2 diabetic patients: systematic review and meta-analysis. BMC Oral Health. 2016;17(1):31-42. https://doi.org/10.1186/s12903-016-0249-1

22. Li Q, Hao S, Fang J, Xie J, Kong XH, Yang JX. Effect of non-surgical periodontal treatment on glycemic control of patients with diabetes: a meta-analysis of randomized controlled trials. Trials. 2015;16(1):291-8. https://doi.org/10.1186/s13063-015-0810-2

23. Engebretson $S$, Kocher T. Evidence that periodontal treatment improves diabetes outcomes: a systematic review and meta-analysis. J Periodontol. 2013;84(4 Suppl):S153-69. https://doi.org/10.1111/icpe.12084.

24. Engebretson SP, Hyman LG, Michalowicz BS, Schoenfeld ER, Gelato MC, Hou W et al. The effect of nonsurgical periodontal therapy on hemoglobin Alc levels in persons with type 2 diabetes and chronic periodontitis: a randomized clinical trial. JAMA. 2013;310(23):2523-32. https://doi.org/10.1001/jama.2013.282431

25. Gay IC, Tran DT, Cavender AC, Weltman R, Chang J, Luckenbach $E$ et al. The effect of periodontal therapy on glycaemic control in a Hispanic population with type 2 diabetes: a randomized controlled trial. J Clin Periodontol. 2014;41(7):673-80. https://doi.org/10.1111/icpe.12268 
26. Simpson TC, Weldon JC, Worthington HV, Needleman I, Wild SH, Moles DR et al. Treatment of periodontal disease for glycaemic control in people with diabetes mellitus. Cochrane Database Syst Rev. 2015;6(11):CD004714. 10.1002/14651858.CD004714.pub3

27. Stephens JM, Lee J, Pilch PF. Tumor necrosis factor- $\alpha$-induced insulin resistance in 3T3-L1 adipocytes is accompanied by a loss of insulin receptor substrate-1 and GLUT4 expression without a loss of insulin receptor-mediated signal transduction. J Biol Chem. 1997;272(2):971-6. https://doi.org/10.1074/jbc.272.2.971

28. Plomgaard P, Bouzakri K, Krogh-Madsen R, Mittendorfer B, Zierath JR, Pedersen BK. Tumor necrosis factor-alpha induces skeletal muscle insulin resistance in healthy human subjects via inhibition of Akt substrate 160 phosphorylation. Diabetes. 2005;54(10):2939-45. https://doi.org/10.2337/diabetes.54.10.2939

29. Stojsavljević S, Gomerčić Palčić M, Virović Jukić L, Smirčić Duvnjak L, Duvnjak M. Adipokines and proinflammatory cytokines, the key mediators in the pathogenesis of nonalcoholic fatty liver disease. World J Gastroenterol. 2014;20(48):1807091. https://doi.org/10.3748/wjg.v20.i48.18070

30. Yazdani-Biuki B, Stelzl H, Brezinschek HP, Hermann J, Mueller T, Krippl $P$ et al. Improvement of insulin sensitivity in insulin resistant subjects during prolonged treatment with the anti-TNF-alpha antibody infliximab. Eur J Clin Invest. 2004;34(9):641-2. https://doi.org/10.1111/j.1365-2362.2004.01390.x

31. Stanley TL, Zanni MV, Johnsen S, Rasheed S, Makimura H, Lee $\mathrm{H}$ et al. TNF-alpha antagonism with etanercept decreases glucose and increases the proportion of high molecular weight adiponectin in obese subjects with features of the metabolic syndrome. J Clin Endocrinol Metab. 2011;96(1):E146-50. https://doi.org/10.1210/¡c.2010-1170

32. Agarwal A, Hegde A, Yadav C, Ahmad A, Manirekar PA, Srikantiah RM. Association of interleukin-6 and myeloperoxidase with insulin resistance in impaired fasting glucose subjects. Indian J Clin Biochem. 2017;32(1):33-8. https://doi.org/10.1007/s12291-016-0567-8

33. Bertoni AG, Burke GL, Owusu JA, Carnethon MR, Vaidya D, Barr RG et al. Inflammation and the incidence of type 2 diabetes: the Multi-Ethnic Study of Atherosclerosis (MESA). Diabetes Care. 2010;33(4):804-10. https://doi.org/10.2337/dc09-1679
34. Kern PA, Ranganathan S, Li C, Wood L, Ranganathan G. Adipose tissue tumor necrosis factor and interleukin-6 expression in human obesity and insulin resistance. Am J Physiol Endocrinol Metab. 2001;280(5):E745-51.

35. Artese HP, Foz AM, Rabelo MS, Gomes GH, Orlandi M, Suvan J et al. Periodontal therapy and systemic inflammation in type 2 diabetes mellitus: a meta-analysis. PLoS One. 2015;10(5):e0128344. https://doi.org/10.1371/journal.pone.0128344

36. D'Aiuto F, Parkar M, Andreou G, Suvan J, Brett PM, Ready D et al. Periodontitis and systemic inflammation: control of the local infection is associated with a reduction in serum inflammatory markers. J Dent Res. 2004;83(2):156-60. https://doi.org/10.1177/154405910408300214

37. Kadowaki T, Yamauchi T. Adiponectin and adiponectin receptors. Endocr Rev. 2005;26(3):439-51. https://doi.org/10.1210/er.2005-0005

38. Tilg H, Moschen AR. Inflammatory mechanisms in the regulation of insulin resistance. Mol Med. 2008;14(3-4):222-31. https://doi.org/10.2119/2007-00119.Tilg

39. Matsumoto S, Ogawa H, Soda S, Hirayama S, Amarasena N, Aizawa $Y$ et al. Effect of antimicrobial periodontal treatment and maintenance on serum adiponectin in type 2 diabetes mellitus. J Clin Periodontol. 2009;36(2):142-8. https://doi.org/10.1111/j.1600-051X.2008.01359.x

40. Sun WL, Chen LL, Zhang SZ, Wu YM, Ren YZ, Qin GM. Inflammatory cytokines, adiponectin, insulin resistance and metabolic control after periodontal intervention in patients with type 2 diabetes and chronic periodontitis. Intern Med. 2011;50(15):1569-74. https://doi.org/10.2169/internalmedicine.50.5166

41. Kharitonenkov A, Shiyanova TL, Koester A, Ford AM, Micanovic R, Galbreath EJ et al. FGF-21 as a novel metabolic regulator. J Clin Invest. 2005 Jun;115(6):1627-35. https://doi.org/10.1172/JCl23606

42. Lin X, Liu YB, Hu H. Metabolic role of fibroblast growth factor 21 in liver, adipose and nervous system tissues. Biomed Rep. 2017;6(5):495-502. https://doi.org/10.3892/br.2017.890 